\title{
Not Recovered or Not Resolved
}

National Cancer Institute

\section{Source}

National Cancer Institute. Not Recovered or Not Resolved. NCI Thesaurus. Code C49494.

One of the possible results of an adverse event outcome that indicates that the event has not improved or recuperated. 